\title{
Complexes of oppositely charged polyelectrolytes and surfactants - recent developments in the field of biologically derived polyelectrolytes
}

\author{
Leonardo Chiappisi, ${ }^{a}$ Ingo Hoffmann ${ }^{\text {ab }}$ and Michael Gradzielski ${ }^{* a}$ \\ We review the work done on complexes between biopolyelectrolytes such as ionically modified cellulose or \\ chitosan and oppositely charged surfactants. Around equimolarity of the charges one typically observes \\ precipitation but for other mixing ratios one may form long-time stable complexes, where structure and \\ rheology depend on the mixing ratio, total concentration and the molecular constitution of the \\ components. In addition, it may be the case that the structures are formed under non-equilibrium \\ situations and therefore depend on the preparation path. The binding is shown to occur cooperatively \\ and the micelles present often retain their shape irrespective of the complexation. However, the rather \\ stiff biopolyelectrolytes may lead to an interconnection between different aggregates thereby forming a \\ network with the corresponding rheological properties. In general, the structure and the properties of \\ the aggregates are rather versatile and correspondingly one can create a wide range of different \\ surfactant-biopolyelectrolyte systems by appropriately choosing the composition. This is very interesting \\ as it allows for formulations with a large range of tuneable properties with ecologically friendly \\ polyelectrolytes for many relevant applications.
}

Received 22nd November 2012 Accepted 11th February 2013

DOI: $10.1039 / \mathrm{cssm} 27698 \mathrm{~h}$

www.rsc.org/softmatter
- the stiffness of the polyelectrolyte chain (for instance characterised by the persistence length)

- the charge density along the polyelectrolyte chain (as controlled by the density of ionisable groups or by $\mathrm{pH}$, e.g. for polyelectrolytes with carboxylic or amine groups).

The phase behaviour of the mixtures and the structures present will depend on these parameters and a generic scheme for the phase behaviour is given in Fig. 1. Typically around equimolarity, i.e., for $Z=1$, there is a pronounced tendency for the formation of precipitates and correspondingly a more or less large two-phase region of precipitated complex and excess water is observed.

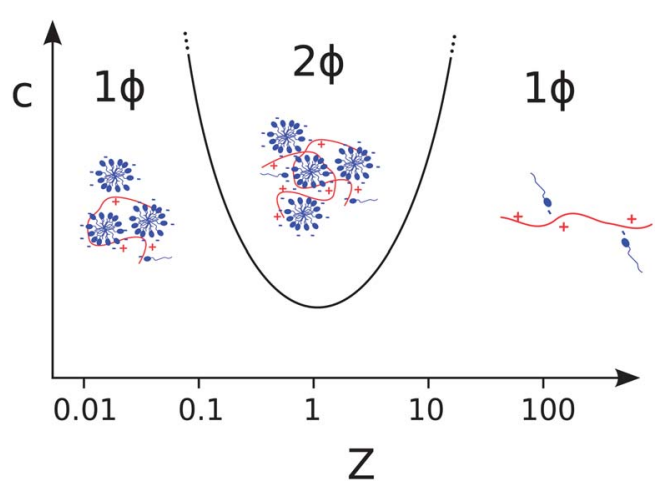

Fig. 1 Schematic phase diagram of oppositely charged surfactant-polyelectrolyte mixtures as a function of the concentration $c$ and the charge ratio $Z=$ [polyelectrolyte]/[surfactant]. 
At $Z>1$ there is an excess of the polyelectrolyte decorated either with individual surfactant molecules or micelles (see also Fig. 2a and c). For $Z<1$ there is an excess of the surfactant and the additional surfactant then simply is able to dissolve the precipitate and stabilise it colloidally. Accordingly here one has a micellar solution in which the polyelectrolyte is fully complexed and stabilized by the surfactant, which means that these complexes carry the charge of the surfactant.

Polymer-surfactant systems are interesting as they are employed in a variety of applications such as detergency, ${ }^{5}$ for drug delivery, ${ }^{6,7}$ as rheological modifiers, ${ }^{\mathbf{8}, 9}$ as delivery systems, ${ }^{10,11}$ in paints ${ }^{12}$ or other formulations. In detergency a relevant question is often their deposition/redeposition onto fabric fibres, a topic that has been studied to quite an extent $\mathbf{1}^{\mathbf{1 3 , 1 4}}$ but shall not be covered here. Accordingly, such mixtures have been investigated for some time, with the main emphasis on synthetic polyelectrolytes such as anionic poly(acrylic acid) (PAA), poly(methacrylic acid) (PMAA), poly(styrene sulfonate) (PSS), or cationic poly(diallyldimethylammonium chloride) (PDADMAC), where the charges are very closely spaced, i.e., the distance between the charges is much less than the Bjerrum length (eqn (1); with $\varepsilon_{\mathrm{r}}$ : dielectric constant, $\varepsilon_{0}$ : permittivity

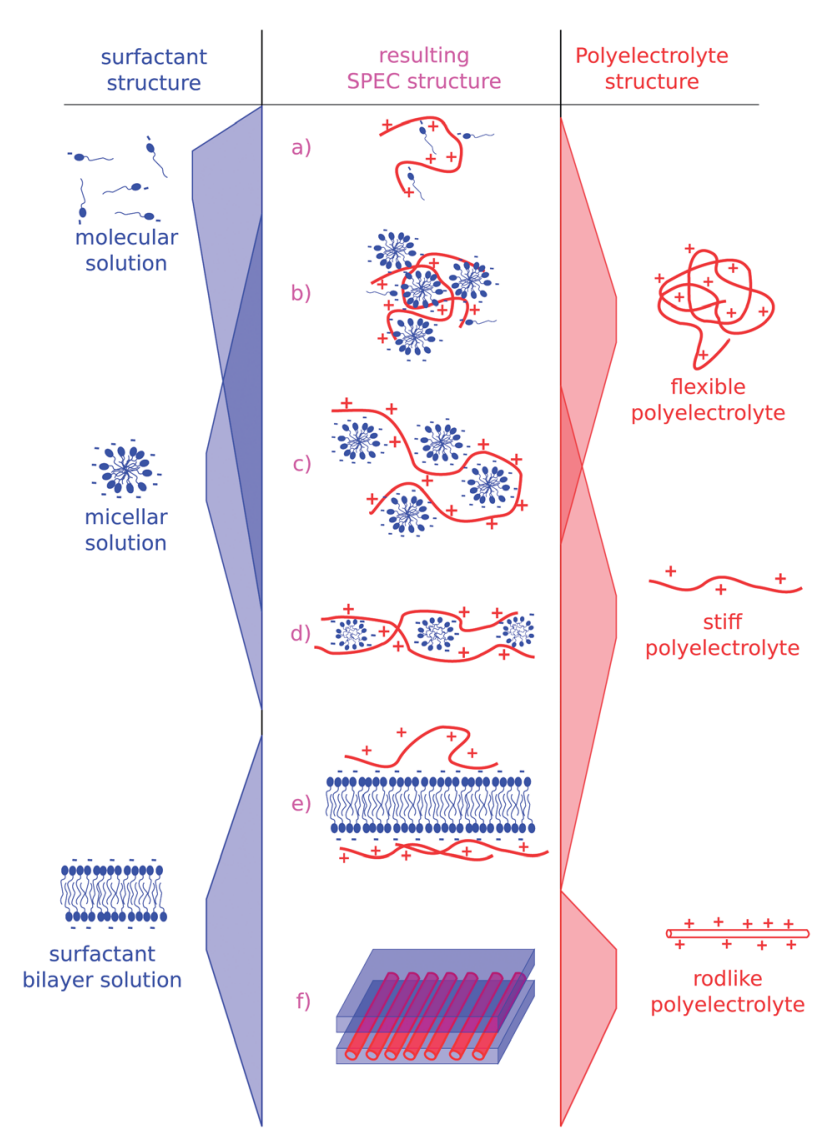

Fig. 2 Schematic description of possible arrangements in surfactant-polyelectrolyte complexes (SPECs): (a) polyelectrolyte decorated with surfactants; (b) densely packed micelles "glued" together by a polyelectrolyte; (c) pearl-necklace structure; (d) rod-like aggregation of micelles with a stiff polyelectrolyte; (e) flexible polyelectrolyte attached to a bilayer; and ( $f$ ) rod-like polyelectrolyte incorporated between bilayers. constant of vacuum, $k$ : Boltzmann constant and $T$ : temperature), which gives the distance at which the electrostatic energy of two elementary charges becomes equal to $k T$, and accordingly counterion condensation is expected to be relevant here. ${ }^{15}$

$$
\lambda_{\mathrm{B}}=\frac{k T}{4 \pi \varepsilon_{\mathrm{r}} \varepsilon_{0}}
$$

In water the Bjerrum length at room temperature is $0.72 \mathrm{~nm}$. The other relevant electrostatic length in polyelectrolyte solutions is the Debye screening length as given by $\left(N_{\mathrm{A}}\right.$ : Avogadro constant and $e$ : elementary charge):

$$
\delta=\sqrt{\frac{\varepsilon_{\mathrm{r}} \varepsilon_{0} k T}{2 N_{\mathrm{A}} e^{2} I}}
$$

which is mainly controlled by the ionic strength $I$ of the solution, where $\delta$ is $9.6 \mathrm{~nm}$ for a $1 \mathrm{mM}$ solution of a $1: 1$ electrolyte. Once the screening length becomes equal to the Bjerrum length, which is the case for an ionic strength of $\sim 180 \mathrm{mM}$ (actually very close to physiological conditions), the screening becomes increasingly dominant and therefore the particular properties of polyelectrolytes compared to those of neutral polymers become increasingly lost. This then is also seen for the formation of interpolyelectrolyte complexes (IPECs) that above ionic strengths of $400-500 \mathrm{mM}$ does not take place anymore ${ }^{3}$ and also leads to the disintegration of well defined micellar IPEC aggregates. ${ }^{16}$ However, the structures formed in polyelectrolyte-surfactant mixtures will also depend on the type and even more on the concentration of the surfactant, for instance, whether the surfactant concentration is above or below the critical micellar concentration (cmc), whether spherical, rodlike or disklike micelles are present, and, of course, on the mixing ratio $Z$, as well as the other parameters mentioned above.

Some possible structural scenarios are depicted in Fig. 2 and for large $Z$ (polyelectrolyte rich) one may either have the situation where single surfactant molecules are attached to a polyelectrolyte chain (Fig. 2a) or form micellar-type aggregates (Fig. 2b and c). This aggregate formation may take place well below the cmc, being induced by the locally enhanced surfactant concentration and the hydrophobic effect as the driving force. However, in order to have a propensity for forming aggregates the stiffness of the polyelectrolyte should not be too high as otherwise a scenario with singly attached surfactant molecules will be favoured. The detailed structure will depend on the balance for the free energy, i.e., what is the loss of free energy due to bending of the polyelectrolyte (for the case of aggregate formation) and restricting it in space (loss of configurational entropy) compared to the free energy involved in the hydrophobic effect. For concentrations above the cmc one may observe similar structures, where the already present micelles now become arranged in the solution due to their binding to the oppositely charged polyelectrolyte.

The micelle-polyelectrolyte complexes may occur in the form of pearl-necklace structures (Fig. 2b), but can also lead to a dense packing of micelles "glued" together (Fig. 2c), which will depend on the charge density of the polyelectrolyte. ${ }^{17}$ For the case of block copolymers with one polyelectrolyte block and another 
water-soluble block (double-hydrophilic copolymers) this leads to the dispersion of such densely packed micelles in a kind of super-aggregate $^{\mathbf{1 8 , 1 9}}$ and may even yield a liquid crystalline type arrangement. ${ }^{20}$ This then obviously requires the persistence length of the polyelectrolyte to be less than the radius of the micelles in order to allow for a dense arrangement of the polyelectrolyte around the micelles, which depends strongly on the curvature of the surfactant aggregates. For polyelectrolytes with a rather high persistence length one may then observe arrangements of the micelles locally along one axis, thereby forming tube-like polyelectrolyte-micelle aggregates (Fig. 2d).

A main difference between most synthetic polyelectrolytes and polysaccharide based biopolyelectrolytes is the persistence length. Many synthetic polyelectrolytes are derived from vinyl based monomers (such as acrylates, methacrylates, styrene sulfonate, etc.) and therefore possess a rather flexible carbon chain as the backbone. In contrast, polysaccharide based polyelectrolytes have a much more rigid backbone (for instance for carboxymethyl cellulose the persistence length has been found to be $\sim 40 \mathrm{~nm})^{21}$ due to their connected sugar units. This constitutes a major difference, to which one has in addition the fact that for the polysaccharide derived polyelectrolytes one has normally a lower charge density along the polymer (due to the larger monomeric unit) and a polymer backbone with many $\mathrm{OH}$ groups. That allows for an additional interaction via $\mathrm{H}$-bonding which can render the whole interaction with other molecules or colloids rather complex and as well modify their interaction with water. So based on the higher rigidity of the backbone and its lower charge density one can conclude that polysaccharide derived biopolyelectrolytes will be substantially weaker in their effective compensation of charges on highly curved interfaces (such as on a micellar surface) and have a tendency for forming elongated aggregates.

For the opposite case of flat surfactant structures, as for membranes, one requires rather stiff polyelectrolytes in order to have an efficient interaction. This has for instance been observed for the case of lamellar phases and DNA, which is a very stiff polyelectrolyte. Accordingly for this case one can observe the incorporation of DNA into lamellar phases thereby even leading to a highly ordered (hexagonal) phase (Fig. 2f). ${ }^{22}$ For not so stiff polyelectrolytes the binding might be weak as here the binding to the surface will lead to a substantial reduction of its configurational entropy. For the case of much more flexible polyelectrolytes one may still have a sufficiently strong interaction to enable binding to a surfactant bilayer, but then one can end up with a bilayer decorated by the polyelectrolyte (Fig. 2e) that thereby still retains a relatively large degree of configurational freedom.

A further degree of freedom in the polymer architecture arises from the fact that one may have branched/hyperbranched polyelectrolytes or polyelectrolyte brushes, which also will form complexes with surfactants. ${ }^{23}$ Another complication of the situation appears if one considers hydrophobically modified polyelectrolytes, which renders the surfactant-polyelectrolyte interactions even more complex. ${ }^{24}$

In the following we will focus on mixtures of surfactants with biopolyelectrolytes, but for a better comparison will start with a brief overview of results on conventional synthetic polyelectrolytes. Subsequently we will concentrate on ionically modified cellulose and chitosan as two rather important biopolyelectrolytes. They are by far not the only interesting biopolyelectrolytes but are two good representative cases of interest in this review article, on which we also have recently been working on. Of course, a general interest in biopolyelectrolytes arises from the fact that they do not only derive from renewable resources but in addition, usually are well biocompatible, which makes them interesting for a large number of applications, such as biomedicine (drug delivery, tissue engineering), food modification and preservation, or wastewater treatment, where biocompatibility is of crucial importance.

\section{The behaviours of different polyelectrolyte-surfactant mixtures - some general remarks}

In general, this is a very vast field and the purpose of this article is not to yield a full coverage of it. Instead we will focus on a few either very general aspects or ones that are related to observations covered in the subsequent sections on modified cellulose and chitosan.

\subsection{Behaviour of typical synthetic polyelectrolytes}

Typical synthetic polyelectrolytes, which are also available in large quantities commercially, are for instance polyacrylic acid (PAA), polymethacrylic acid (PMAA), and polystyrene sulfonate (PSS). They have in common a $\mathrm{C}_{2}$ repeat unit along the polymer backbone, which means a spacing of the charges of $0.25 \mathrm{~nm}$, which is well below the Bjerrum length defined in eqn (1). In the ionic form they are very well soluble in water but when fully protonated PAA and PMAA lose their water solubility.

Classical cases of polyelectrolyte-surfactant complexes are those of poly(styrene sulfonate) PSS and alkyltrimethylammonium surfactants. Here it has been found that the surfactant aggregates contained in the complexes possess aggregation numbers that are independent of the surfactant concentration and the type of counterions and increase with the length of the alkyl chain of the surfactant in the conventional way observed for free surfactant micelles. ${ }^{25}$ This means that structurally the micelles are not much affected by the presence of the polyelectrolyte, which then is only decorating the micelles. Such behaviour is quite frequently observed in these complexes.

Another much investigated system is poly(acrylic acid) (PAA), respectively its sodium salt (NaPA), for instance in mixtures with alkyltrimethylammonium bromide $\left(\mathrm{C}_{n} \mathrm{TAB}\right)$. Due to the high charge density along the NaPA backbone one observes a rather large 2-phase region ( $c f$. Fig. 1) but its extent depends strongly on the length of the alkyl chain of the $\mathrm{C}_{n} \mathrm{TAB}$. For a given NaPA concentration, it becomes smaller with increasing monomeric solubility of the surfactant, i.e., with decreasing surfactant chain length. ${ }^{26}$ Furthermore it was observed that this phase separation can be suppressed by addition of simple salts, or in other words the complex is salted-in. More recent investigations have addressed this salt effect in more detail for the 
system PSS- $\mathrm{C}_{16}$ TAB and the influence of the addition of $\mathrm{NaCl}^{27}$ In this study it was observed that low $\mathrm{NaCl}$ concentrations mostly reduce the composition range in which kinetically trapped aggregates are observed, while high $\mathrm{NaCl}$ concentrations affect the equilibrium phase behaviour by reducing the amount of the surfactant bound to the polyelectrolyte.

The addition of a surfactant to a polyelectrolyte typically also affects the viscosity and for the case of high molecular weight (750 $\mathrm{kDa}$ PAA the addition of excess cetyltrimethyl ammonium bromide $\left(\mathrm{C}_{16} \mathrm{TAB}\right)$ has been observed to lead to a reduction of viscosity and to a disappearance of the shear-thinning behaviour of the pure PAA solutions. ${ }^{28}$ Such a reduction of viscosity then is typically related to a compaction of the polyelectrolyte by the oppositely charged surfactant thereby reducing its effective hydrodynamic volume. Such lowered viscosity has also been observed in mixtures of PAA and $\mathrm{C}_{12} \mathrm{TAB}$ and was explained by a quantitative model based on two effects: a shortening of the effective chain length of the polyelectrolyte (due to the wrapping around the micelles) and the release of salt by combining the surfactant and the polyelectrolyte. ${ }^{29}$ However, for lower surfactant addition $(Z<1)$ in the semidilute regime both $\mathrm{C}_{12}$ TAB and $\mathrm{C}_{16} \mathrm{TAB}$ were found to lead to a viscosity increase which was attributed to a bridging of the polyelectrolyte by the surfactant micelles. ${ }^{30}$

\subsection{Ordered structures in complexes}

The structure of complexes formed in the system SDS-poly-2(propionyloxy)ethyl-trimethylammonium chloride (PCMA) has been studied to some extent by small-angle neutron scattering (SANS), which by employing deuterated SDS allows to deduce detailed structural information. These experiments showed that already the addition of relatively small amounts of the surfactant leads to a substantial shift of the typical correlation peak of the polyelectrolyte. Once a ratio $Z=3$ is reached a prominent peak corresponding to a spacing of 3.7-3.9 $\mathrm{nm}$ is observed, which arises from the correlation of densely packed micelles. ${ }^{31}$ Such highly ordered arrangements of micelles within the surfactant-polyelectrolyte complex (Fig. 2b) are a very interesting aspect and have been studied intensely over the last few years. This structure has for instance been reported for copolymers of poly(sodium methacrylate- $c o-N$-isopropylacrylamide) (p(MANaco-NIPAM)) where, depending on the ratio of the two monomers contained in the copolymer (thereby controlling the charge density along the polyelectrolyte backbone), upon admixture of $\mathrm{C}_{16} \mathrm{TAB}$ highly ordered phases are observed. For the case of 100 $\mathrm{mol} \%$ (of the anionic copolymer) PMANa a Pm3n structure, for 67 mol\% PMANa a fcc structure and for 50 mol\% PMANa a hcps structure was found, ${ }^{32}$ which means that the local ordering of the same type of micelles depends strongly on the charge density along the backbone of the polyelectrolyte.

This effect of the charge density on the structure of polyelectrolyte-surfactant complexes has also been addressed in a systematic fashion for a copolyelectrolyte composed of cationic 3-(2-methylpropionamido)propyltrimethyl-ammonium chloride (MAPTAC) and uncharged acrylamide (AM) in mixtures with SDS, where by altering the composition of the initial monomer mixture one can control the charge density of the final polyelectrolyte. These experiments showed that the range of precipitate formation increases systematically with increasing charge density of the polyelectrolyte and in addition their formation is more facile for a more flexible polyelectrolyte. ${ }^{33}$ It is also observed that the ability to form highly ordered micellepolyelectrolyte complexes becomes significantly reduced upon lowering the charge density of the polyelectrolyte.

\subsection{Binding isotherms}

Binding isotherms of surfactants to polyelectrolytes can often be described by the Yang-Satake model, ${ }^{34}$ based on the ZimmBragg theory, ${ }^{35}$ where the fractional saturation, i.e., the fraction of polyelectrolyte units which bind a surfactant molecule, is described as: ${ }^{34}$

$$
\beta=\frac{1}{2}\left[1+\frac{K \omega C_{\mathrm{s}}-1}{\sqrt{\left(1-K \omega C_{\mathrm{s}}\right)^{2}+4 K C_{\mathrm{s}}}}\right] .
$$

Here $K$ is the equilibrium constant for the binding of a single surfactant molecule to a binding site, $\omega$ is the cooperativity parameter and $K \omega$ is the equilibrium constant for the binding to a site adjacent to an already occupied one. A schematic representation of the model is given in Fig. 3.

However, even if the model gives a simple view of the binding process which can be applied to compare the binding strength in different surfactant polymer mixtures, it is an oversimplification of the complex formation. For instance the model does not consider steric hindrance or interactions between surfactant tails across non-occupied sites, ${ }^{36,37}$ it does not consider interactions with more than one neighbour and, finally, as the polymer backbone is not a rigid linear array of sites, it is likely that bound surfactant molecules can interact with each other even if they are located at distant positions via polymer loops.

However, despite these numerous assumptions, the model has been shown to be able to describe the binding isotherms in very different systems. ${ }^{38-46}$ The most common ways for the determination of the binding isotherms are potentiometric ${ }^{38-43}$ and calorimetric titrations, ${ }^{\mathbf{4 4 - 4 6}}$ with the latter having the advantage that also the binding enthalpies can be obtained, therefore resulting in a comprehensive thermodynamic characterization of the binding process.

As stated above, the focus of this review shall be on surfactants with oppositely charged biopolyelectrolytes, a topic that has been evolving strongly during recent years. ${ }^{47-49}$ This is driven by the availability of such polyelectrolytes from renewable resources and that they are generally ecologically friendly

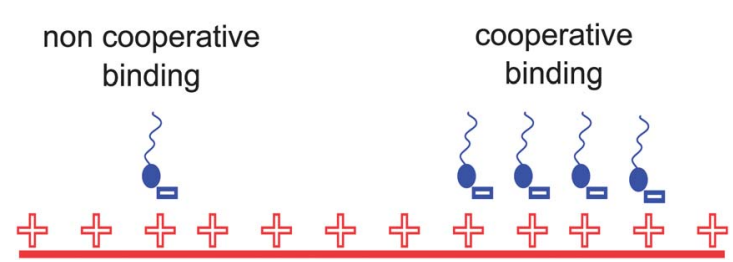

Fig. 3 Schematic representation of the binding of surfactants to an oppositely charged polyelectrolyte. 
and therefore well suited for a large number of applications. In the following we will in particular discuss work on ionically modified cellulose and chitosan as interesting examples of biopolyelectrolytes. We will not just focus on structure and phase behaviour of these compounds but also cover exemplarily various physico-chemical aspects of surfactant-polyelectrolyte complexes, such as their interfacial aspects (Sections 3.5 and 4.3) or the thermodynamics of binding (Sections 3.4 and 4.2).

Of course, chitosan and cationically modified cellulose are not the only biopolyelectrolytes of interest and it might be noted that there are a number of other biopolyelectrolytes, such as hyaluronic acid, chondroitin sulfate, dermatan sulfate, keratan sulfate, etc. which also have been investigated to some extent in recent times. And, of course, one has DNA as a model example of a stiff biopolyelectrolyte that has been studied extensively in combination with surfactants and this applies similarly to proteins, which depending on $\mathrm{pH}$ also have some polyelectrolyte properties. However, all of the latter will not be considered in this review as that would render the field to be covered by far too large.

\section{Cellulose based polyelectrolytes and oppositely charged surfactants}

\subsection{Types of cellulose based polyelectrolytes and their synthesis}

Cellulose is a one of the most abundantly available biopolymers. It constitutes $50 \mathrm{wt} \%$ of plants' cell walls. Its monomers are glucose molecules connected by $\beta$-1,4-glycosidic bonds (see Fig. 4). The dimer of two such glucose molecules is referred to as cellobiose and can also be considered as the monomeric unit of cellulose and typically, the molecular weight is on the order of some $100000 \mathrm{~g} \mathrm{~mol}^{-1}$. While natural cellulose is uncharged and insoluble in water, there are numerous functionalized, water soluble cellulose polymers available, ${ }^{50,51}$ several polyelectrolytes among them. Usually the three hydroxyl groups serve as reaction sites to attach different functional groups via an ether bond. Therefore, every monomeric unit can be functionalized up to three times and the extent to which the cellulose is functionalized is usually expressed as the degree of substitution (DS), giving the average number of hydroxyl groups exchanged by a different functional group per monomeric unit, thus ranging from 0 to 3 .

As an alternative to functionalization via an ether bond, it is also possible to obtain deoxy amino cellulose derivatives ${ }^{52,53}$ which are synthesized via tosylated cellulose $\mathrm{e}^{54}$ or cellulose esters. ${ }^{55}$ Recently, zwitterionic amino cellulose sulfate derivatives

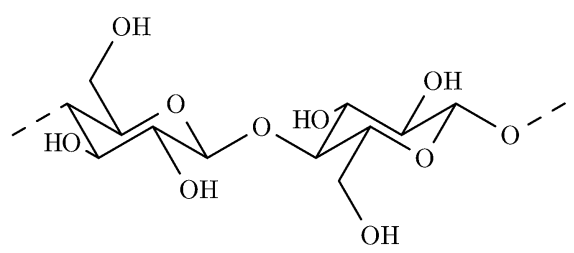

Fig. 4 Chemical structure of cellulose. have been obtained. ${ }^{56,57}$ While the most common usage for cellulose is in paper production, cellulose derivatives are used as anti-redeposition agents in detergent formulations, as conditioners in shampoos or as thickeners, ${ }^{58-60}$ not least because cellulose is a renewable resource and the material is biocompatible and biodegradable. The structure and dynamics in bulk and at the interface of complexes formed from oppositely charged cellulose based polyelectrolytes and surfactants have been studied extensively over the past few decades and several review articles dealing with different aspects of the use of water soluble cellulose based polymers are available. ${ }^{5,61-69}$ Among the available functionalised cellulose based polyelectrolytes, the most commonly used ones are carboxymethylcellulose (CMC) as a polyanion and hydroxyethylcellulose with an additional glycidyltrimethyl-ammonium chloride functionality as a polycation (catHEC), which is commercially available as JR 400 (other polymers with different molecular weights and DS are e.g. JR 125, JR 30M, LR 400 or LR 30M, where the LR polyelectrolytes have a lower DS than the JR polyelectrolytes). By replacing one of the methyl groups attached to the nitrogen by a longer chain, the properties of a polycation and a hydrophobic polymer can be combined (Fig. 5). Such a polymer with a chain of 12 carbon atoms is available under the name LM 200. Similar other polymers with different molecular weights and DS have been investigated, where it might be noted that in general cellulose based polymers have a rather large persistence length, i.e., are rather stiff polymers. In the following a concise overview of this work is given.

\subsection{Phase behaviour}

A considerable number of publications deals with the phase behaviour of oppositely charged cellulose based polyelectrolyte-surfactant systems.,.$^{5,44,63,70-79}$ As for other polyelectrolytes, the phase behaviour of mixtures of cellulose based

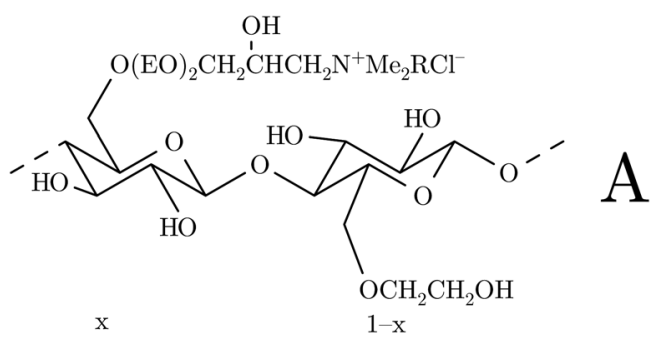

JR 400: $\mathrm{R}=\mathrm{CH}_{3}$ LM 200: $\mathrm{R}=\mathrm{C}_{12} \mathrm{H}_{25}$
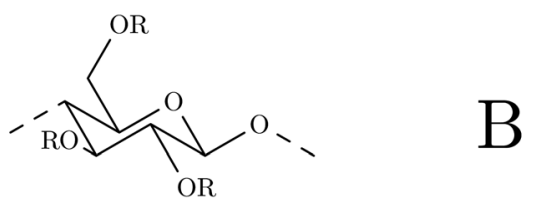

$\mathrm{CMC} \mathrm{R}=\mathrm{H}, \mathrm{CH}_{2} \mathrm{COO}^{-} \mathrm{Na}^{+}$

Fig. 5 (A) Chemical structure of cationically and hydrophobically and cationically modified hydroxyethylcellulose. For JR $400 x=0.27$ and the molecular weight is $500000 \mathrm{~g} \mathrm{~mol}^{-1}$, for LM $200 x=0.03$ and $M_{\mathrm{w}}=100000 \mathrm{~g} \mathrm{~mol}^{-1}$. (B) Chemical structure of $C M C$. 
polyelectrolytes and oppositely charged surfactants is characterized by two homogeneous single phase regions with an excess of either polyelectrolyte or surfactant. These phases are separated by a two phase region around equal polyelectrolyte and surfactant charges, in which a neutral complex precipitates (Fig. 1). ${ }^{5,70-74}$ It has been found that these complexes can be dissolved in an excess of polyelectrolyte or surfactant and their net charge has been reported to change sign in the case of JR 400-SDS, JR 400-SDBS and CMC-CTAB ${ }^{14,44,80}$ but not in the case of CMC-DTAB, ${ }^{80}$ for which no explanation has been advanced, yet. For mixtures of other cationic surfactants of the 1-alkyl-3-methylimidazolium bromide and $N$-alkyl- $N$-methylpyrrolidinium bromide type with CMC it has been shown that the earlier the region of the phase separation is reached the longer is the alkyl chain of the surfactant. ${ }^{81}$ At very low concentrations, the situation can be somewhat different ${ }^{75-78}$ and some deviations are observed with different surfactants, probably due to different critical aggregation concentrations (cac) of different surfactants. For the cationic JR 400 and SDS some contradicting results can be found in the literature concerning the surfactant concentration necessary to redissolve the precipitates at low JR 400 concentrations. On the one hand, Goddard and Hannan ${ }^{70}$ reported a two phase region reaching considerably beyond the charge equilibrium, while Yamaguchi et al. found a phase boundary in good agreement with it. ${ }^{79}$ Recently, Li et al. ${ }^{44}$ could show with the help of ITC measurements that this discrepancy is due to the formation of meta-stable aggregates, the life span of which depends on the route of preparation. This is in general an important point in surfactant-polyelectrolyte mixtures, where it is not easily guaranteed to achieve an equilibrium situation.

Zhou et al. ${ }^{72}$ investigated the phase behaviour of mixtures of cat-HECs of different molecular weights and different numbers of ethylene oxide (EO) groups between the backbone and the charge. Polyelectrolytes with a higher molecular weight tend to precipitate at lower surfactant concentration and a larger number of EO groups tends to prevent precipitation. The phase boundary on the surfactant excess side does not seem to be influenced much by the molecular weight or the number of EOs. Sjöström et al. ${ }^{82}$ investigated the swelling and collapse of gels, prepared from covalently cross-linking JR 400 with DVS (divinyl sulfone), upon addition of salt and SDS. The collapse of the gels can be related to the precipitation that is observed in solutions of non-cross-linked polyelectrolytes and SDS and it is observed that addition of $\mathrm{NaCl}$ shifts the collapse to higher SDS concentrations. However, at a salt concentration of $1 \mathrm{M}$, a lower SDS concentration was necessary again. The reswelling, which can be related to the redissolution phase boundary, is shifted to lower SDS concentrations upon addition of the salt.

\subsection{Bulk properties}

Comprehensive work has dealt with the change of viscosity in oppositely charged cellulose based polyelectrolyte-surfactant systems and hydrophobically modified cellulose based polymer-surfactant systems. ${ }^{71,72,78,83-88}$
At rather low polyelectrolyte concentrations, the viscosity is decreased upon addition of an oppositely charged surfactant. This is due to the collapse of the polyelectrolyte chains, when their charges are compensated by the surfactant ${ }^{83,86}$ and thereby one then has a reduced effectively hydrated volume. Therefore, such mixtures may be employed as rheological modifiers in formulations, again mostly for the reason of having a biocompatible polyelectrolyte.

The situation becomes more complex in the semi-dilute concentration range. It has been observed many times that hydrophobically modified polymers can form networks based on hydrophobic contact points resulting in increased viscosities compared to their unmodified analogues. ${ }^{71,87}$ Adding surfactants to such networks leads to a very pronounced increase in viscosity, often by several orders of magnitude, ${ }^{69,71,78,87,89}$ by rendering the contact points more long-lived and/or frequent. A similar behaviour can be observed for the addition of oppositely charged surfactants to polyelectrolytes and several such experiments have been carried out with cellulose based polyelectrolytes. ${ }^{71,76,77,90}$ Initially, a somewhat higher viscosity as compared to water is observed due to the polyelectrolyte (see Fig. 6a and 2a for the case of JR 400 as the polyelectrolyte). Beyond a certain onset surfactant concentration a further increase in viscosity is observed. Initially, individual surfactant molecules bind electrostatically to the polyelectrolyte backbone (Fig. 6b). At the onset concentration, aggregate formation on the backbone starts and several chains become interconnected, leading to an enhanced viscosity, which reaches a maximum next to the phase boundary (Fig. 6c), beyond which precipitation sets in. For high enough surfactant concentration the precipitate becomes resolubilised and then the aggregates are no longer dominated by the polyelectrolyte but by the surfactant and the aggregates no longer sufficiently interconnect the chains, which results in a drop of viscosity down to the level of the pure polyelectrolyte solution and even below (Fig. 6d) and here the charges of the polyelectrolyte are fully saturated by the surfactant.

Dynamic light scattering (DLS) $72,76,85$ measurements have been carried out on such viscoelastic networks to examine their dynamics. It has been found that the field autocorrelation

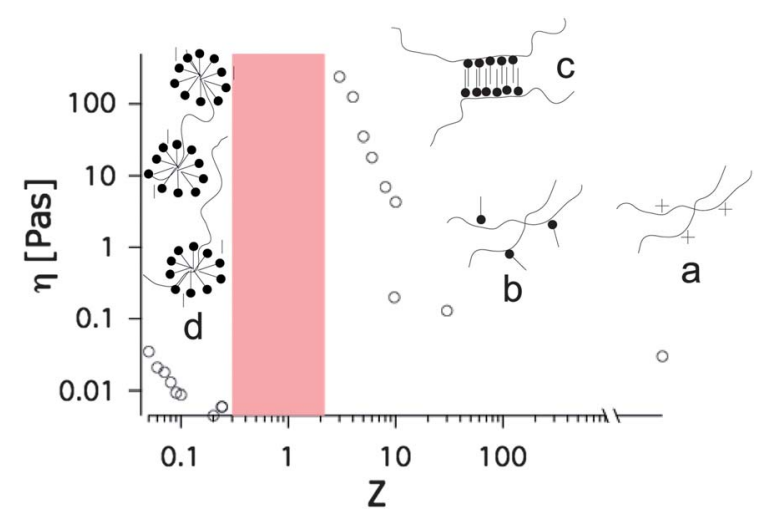

Fig. 6 Viscosity of a 1 wt\% solution of JR 400 with added SDBS as a function of charge ratio; see the text for details. 
function shows two decays: a fast single exponential decay, behaving diffusively, and a slower stretched exponential decay, which is non-diffusive:

$$
g^{(1)}(t)=A_{\text {fast }} \exp \left(-t / \tau_{\text {fast }}\right)+A_{\text {slow }} \exp \left(-\left(t / \tau_{\text {slow }}\right)^{\beta}\right)
$$

While the slow mode $\tau_{\text {slow }}$ becomes increasingly slow as more surfactant is added until the precipitation threshold, the fast mode $\tau_{\text {fast }}$ is almost constant with composition. This sort of behaviour is predicted in the framework described by Ngai et al. ${ }^{91-93}$ This mode coupling theory (MCT) approach has been successfully applied to describe the dynamics of semi-dilute polymer solutions. ${ }^{94-96}$ While the decay at short times, described by $\tau_{\text {fast }}$, is related to the collective motion of single chain segments with the diffusion coefficient $D=\left(\tau_{\text {fast }} Q^{2}\right)^{-1}$, where $Q$ is the scattering vector, the slow mode, described by $\tau_{\text {slow }}$, accounts for the dynamics of larger clusters in the network. The exponent of the stretched exponential is related to the coupling parameter $n$ via $\beta=1-n$. The theory assumes that below a certain critical time $t_{\text {crit }}$, the dynamics of the chains are not perturbed by the formation of clusters and the correlation function can be described by a single exponential decay $g(t)=$ $\exp \left(-t / \tau_{\text {fast }}\right)$. For times longer than $t_{\text {crit }}$ the dynamics of the clusters start to influence the correlation function and a second slow mode is required to describe its behaviour. The decay time of the slow mode is related to $\tau_{\text {fast }}, t_{\text {crit }}$ and $n$ as $\tau_{\text {slow }}(Q)=$ $\left(t_{\text {crit }}{ }^{-n} \tau_{\text {fast }}(Q)\right)^{1 /(1-n)}$. While the fast time shows diffusive behaviour i.e., $\tau_{\text {fast }} \propto Q^{-2}$, the $Q$ dependence of $\tau_{\text {slow }}$ is proportional to $Q^{-2 /(1-n)}$, as follows from inserting the $Q^{-2}$ dependence of $\tau_{\text {fast }}$ into the expression for $\tau_{\text {slow }}$.

It should be noted that such behaviour is not limited to oppositely charged polyelectrolyte surfactant mixtures but can also be observed in pure semi-dilute polymer solutions or mixtures of nonionic polymers and surfactants or hydrophobically modified polymers and surfactants..$^{\mathbf{6 9 , 8 4 , 8 5 , 9 7 - 9 9}}$

Rheology has been shown to yield qualitatively similar results. Oscillatory rheology shows a cross-over between $G^{\prime}$ and $G^{\prime \prime}$ shifted to lower frequencies as more surfactant is added and steady shear rheology shows shear thinning behaviour with a critical shear rate shifted to lower shear rates upon addition of the surfactant, indicating a slower relaxation time,,$^{71,76,77,87,88}$ as seen by DLS.

Some investigations of the structure of viscoelastic networks consisting of cellulose based polyelectrolytes and oppositely charged surfactants have been carried out with small angle neutron scattering (SANS). It has been found that a number of anionic surfactants form junctions in the shape of rodlike aggregates in systems with cationic hydroxyethyl cellulose JR 400 (ref. 76 and 77) and locally stretched structures have also been observed for negatively charged hydroxyethylcellulose derivatives and CTAB as well as for cationically modified starch $^{\mathbf{1 0 0}}$ and PDADMAC with SDS. ${ }^{\mathbf{1 0 1}}$ At lower scattering vector another upturn becomes visible which disappears in the surfactant excess region $(Z<1)$, and the viscosity is lowered again. Using hydrogenated polyelectrolytes and surfactants, mostly surfactant micelles are visible but by using deuterated surfactants it is possible to only observe the polyelectrolyte and it can be seen that it still has its elongated structure (see Fig. 7). This means that the local structure of the polyelectrolyte is not changed but it is not incorporated in a network anymore, as it had connected the polyelectrolyte chains before at lower $Z$. The structure then is similar to that observed on the polyelectrolyte rich side $(Z=10)$ of these mixtures (see Fig. 7). This is also supported by TEM images ${ }^{\mathbf{1 0 2}}$ on a similar cat-HEC-SDBS system, where the collapse of the network can be observed. It should be noted that the formation of rod-like aggregates is not a general feature. The shape of the aggregates depends on various parameters as it does in pure surfactant solutions. By changing the size of the surfactant aggregates, the rheological behaviour can be influenced, since larger aggregates will generally crosslink more polyelectrolyte chains, thereby leading to higher shear moduli. ${ }^{\mathbf{6 5 1 0 3}}$ Generally, the original form of surfactant aggregation (without polyelectrolyte) often tends to dictate the structure of the surfactant in the complexes in the one phase region. However, the structure of the surfactant in the precipitates depends on the linear charge density. While high charge density synthetic polymers promote crystallinity, this is not as much observed in precipitates with cellulose based polyelectrolytes, which generally have a lower charge density.

At low polyelectrolyte concentrations, it has been found that relatively well defined and monodisperse particles can be formed. Langevin et al. ${ }^{\mathbf{8 3 , 1 0 4}}$ investigated mixtures of CMC (DS = 0.7-1.23) and DTAB and found that monodisperse particles were formed above the cac, despite using a polydisperse polymer. The size of these particles could be controlled by the surfactant concentration over a range from some 10 to some 100 nanometers and SANS measurements showed that they had a liquid crystalline interior of densely packed DTAB micelles as confirmed by SANS contrast variation experiments ${ }^{75}$ ( $c f$. Fig. 2b).

Another interesting application of cellulose based polyelectrolyte-surfactant systems is the formation of mm-sized capsules, which can be easily obtained by pipetting a solution of

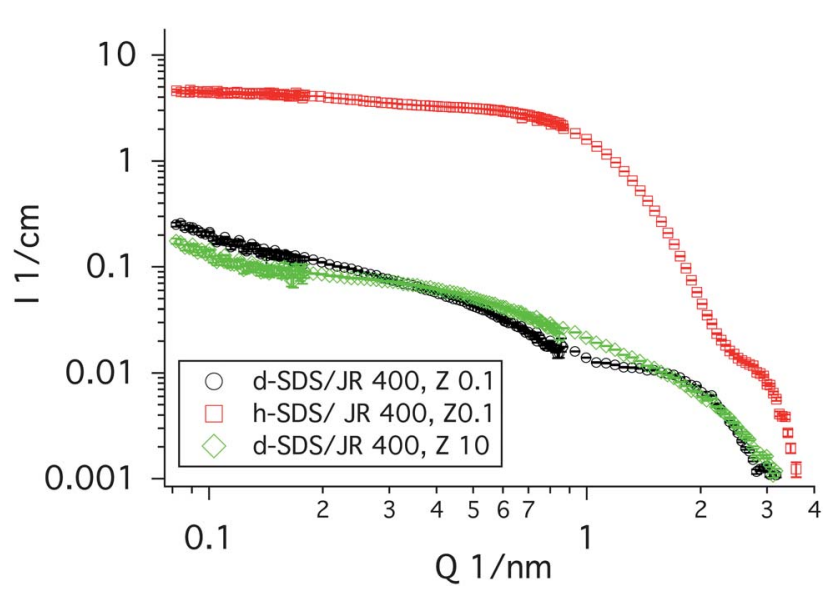

Fig. 7 SANS curves of JR 400 (1 wt\%) with deuterated SDS (d-SDS, black circles, only polyelectrolyte visible) and hydrogenated SDS (h-SDS) in deuterated water each with a tenfold excess of surfactant charges and d-SDS with 1 wt\% JR 400 with a tenfold excess of PE charges (green diamonds). The $Q^{-1}$ slope in the curves with deuterated surfactant indicates the elongated structure of the polyelectrolyte in both PE and surfactant excess. 
a surfactant into a solution of a polyelectrolyte (or vice versa), so that the local concentration is in the precipitation region of the phase diagram. Here the large $M_{\mathrm{w}}$ and the stiffness of the polymer might aid in stabilizing the shell of the formed capsules. By adding a third component to the solution, which is pipetted into the other solution, these capsules can serve as a container for the third component. Lapitsky and Kaler used JR 400 and SDS to obtain such capsules and used them for the encapsulation of oil droplets ${ }^{\mathbf{1 0 5}}$ and also studied effects of chemical cross-linking on the reversible swelling with surfactant and its release. ${ }^{\mathbf{1 0 6}}$

Fluorescence methods can yield useful information about polymeric systems. ${ }^{107}$ Polysaccharide based polymers are well suited for the use of fluorescence methods, since they are easy to label ${ }^{\mathbf{1 0 8 , 1 0 9}}$ and a number of studies has been performed with labeled cellulose derivatives ${ }^{\mathbf{1 4 , 6 1 , 1 1 0 - 1 1 2}}$ and also with non-covalently attached fluorescence labels. ${ }^{\mathbf{1 0 2}, \mathbf{1 1 3}}$ Most noticeably the formation of hydrophobic surfactant aggregates along the polyelectrolyte backbone and conformational changes of hydrophobically modified cationic cellulose could be shown by the change of the fluorescence signal. ${ }^{61,102,110}$

\subsection{Surfactant binding isotherms}

Adsorption isotherms of ionic surfactants to oppositely charged, cellulose based polyelectrolytes have been investigated using a number of methods, for example dialysis, ${ }^{114}$ potentiometry $^{\mathbf{8 6}}$ and isothermal titration calorimetry (ITC). ${ }^{\mathbf{4 4 1 1 5}}$ Sjöström and Piculell used the swelling of covalently crosslinked gels as an easy to use alternative to the aforementioned methods. ${ }^{82}$

Mostly cat-HEC-SDS ${ }^{44,82,114}$ but also CMC-DTAB ${ }^{86}$ and catHEC-sodium perfluorooctanoate have been investigated. ${ }^{\mathbf{1 1 5}}$ The Yang-Satake model (eqn (3)) has been successfully applied to describe the obtained binding isotherms in the low surfactant concentration region of cat-HEC-anionic surfactant systems and CMC-DTAB, while it fails to describe the binding isotherms obtained with PSS-DTAB; this has been attributed to the fairly large monomeric units in cellulose based polyelectrolytes, making steric aspects, not taken into account in the model, negligible. ${ }^{\mathbf{1 1 5}}$

It is generally observed that initially the surfactant is bound in a non-cooperative manner. Subsequently, a critical aggregation concentration (cac) cooperative binding is observed until phase separation. On adding sufficient surfactant, the precipitates are redissolved. This redissolution is attributed to the formation of mixed aggregates stabilized by polyelectrolyte bound surfactant micelles. ${ }^{\mathbf{4 4}}$

\subsection{Interfacial aspects}

Many studies investigate effects of polyelectrolyte-surfactant mixtures on the surface tension and some of them have been carried out with cellulose based polyelectrolytes and oppositely charged surfactants, ${ }^{5,66-68,70,71,83,86,104,116,117}$ as such systems are for instance very important in detergency. The general picture is similar to that obtained with other polyelectrolytes (see Fig. 8). The surface tension as a function of surfactant concentration at

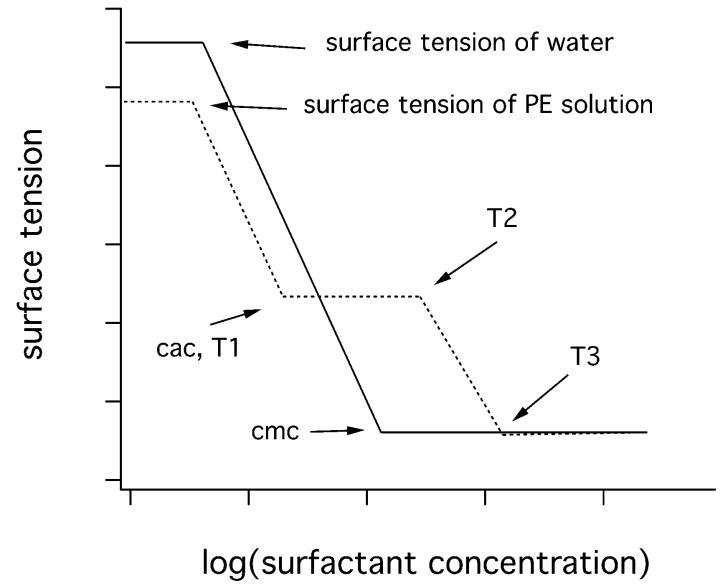

Fig. 8 Idealised surface tension plot for both mixtures (dashed line) and pure surfactant (straight line). The formation of PE bound surfactant aggregates starts at $\mathrm{cac} / T_{1}$; at $T_{2}$ the PE is saturated and the formation of free surfactant micelles starts at $T_{3}$.

a constant polyelectrolyte concentration starts decreasing upon addition of the surfactant and reaches a plateau at a concentration usually designated cac (critical aggregation concentration or $T_{1}$ ) which is usually significantly lower than the cmc (critical micellar concentration). After the plateau, a second decrease is observed (cac2 or $T_{2}$ ), which is followed by another plateau, the onset of which is the cmc $\left(T_{3}\right)$. This is usually interpreted in a way that surface active polyelectrolyte-surfactant aggregates are formed at the cac, while at the cac2 the polyelectrolyte is saturated and eventually the mixed aggregates are displaced from the interface by the surfactant. This is also supported by measurements of the surface viscoelasticity, ${ }^{\mathbf{1 1 8 , 1 1 9}}$ where it has been found that the interface becomes viscoelastic upon addition of the surfactant, which means that polyelectrolyte-surfactant complexes are adsorbed at the interface. At higher surfactant concentration, the viscoelasticity is lost, which means that the aggregates are displaced from the surface by the surfactant.

While surface tension gives only indirect evidence for the formation of mixed aggregates, other methods like ellipsometry or reflectometry allow the measurement of thickness and structure of polyelectrolyte-surfactant aggregates at interfaces and a large body of data has been accumulated over the past decade, a number of them dealing with cellulose based polyelectrolytes. ${ }^{\mathbf{1 2 , 6 8 , 1 2 0 - 1 2 7}}$

Zimin et al. ${ }^{\mathbf{1 2 0 , 1 2 1}}$ studied the adsorption and desorption of premixed JR 400-SDS complexes on several solid surfaces by AFM and found that the changes in the structure of aggregates at the interface were mainly determined by the bulk structure of the complexes. The adsorption of several cationically modified cellulose based polyelectrolytes and cationically and hydrophobically modified cellulose based polyelectrolytes on silica and hydrophobized silica was studied by Terada et al. with ellipsometry. ${ }^{12,122,123}$ It was found that the amount adsorbed at a given polyelectrolyte concentration with variable SDS concentration is maximal near the phase boundary. Increasing the SDS concentration beyond the two-phase region reduces the amount adsorbed. Interestingly, the thickness of the layer is more or less 
constant before the phase boundary (i.e., at low surfactant concentrations) but increases after the phase boundary. This means that the layer becomes increasingly compact before the phase boundary. After the phase boundary, the complexes are bound more loosely since they now bare a net negative charge on the negatively charged silica surface. Upon rinsing, the adsorbed amount increased. This was interpreted as being due to reduced screening by counterions which are washed away. The adsorbed amount was generally higher on hydrophobized silica and the amount adsorbed started to increase at significantly lower SDS concentrations on hydrophobized silica for cationically modified cellulose. Svensson et al..$^{\mathbf{1 2 4 , 1 2 5}}$ studied the adsorption of mixtures of cationically modified cellulose-SDS and cationically modified guar-SDS and also showed that such systems can be used to deliver silicone oil to negatively charged surfaces.

Some studies deal with the adsorption of cellulose based polyelectrolytes and oppositely charged surfactants on cotton. ${ }^{\mathbf{1 4 , 1 2 8}}$ It is interesting to note that no peak in adsorption was observed near the phase boundary by Hoffmann et al. ${ }^{14}$ for cationically modified cellulose and SDBS as it has been observed on flat model surfaces with SDS as the surfactant. This may be due to the ability of the polyelectrolyte to penetrate into the structure of the negatively charged fibre, as has been observed for cotton ${ }^{129}$ and hair. ${ }^{112}$

\section{Chitosan}

Chitin, poly- $\beta-1-4-N$-acetyl-D-glucosamine, is after cellulose the second most abundant polymer in nature. It is a main structural component of the exoskeleton of crustaceans and insects and is commonly found in the cell wall of fungi. Chitin is insoluble in all common solvents, but it (and also cellulose) can be dissolved in ionic liquids based on imidazolium salts ${ }^{\mathbf{1 3 0}}$ and in dimethylacetamide (DMA) in the presence of $\mathrm{LiCl}^{131}$ Chitosan, a random copolymer of $N$-acetyl-D-glucosamine and D-glucosamine (see Fig. 9) is soluble in aqueous solutions of acids, such as formic and acetic acids due to protonation which renders chitosan a polycation at low $\mathrm{pH}$. Chitosan itself, however, occurs rarely in nature and is obtained by alkaline or enzymatic deacetylation of chitin. Due to its ease of modification, its high availability and low cost, and its biocompatibility, chitosan is employed in a variety of fields. Therefore a large number of papers dealing with practical applications of chitosan have appeared in the last thirty years. Accordingly, depending on the field of application several reviews can be found in the literature. Beside books, book chapters and reviews dealing with

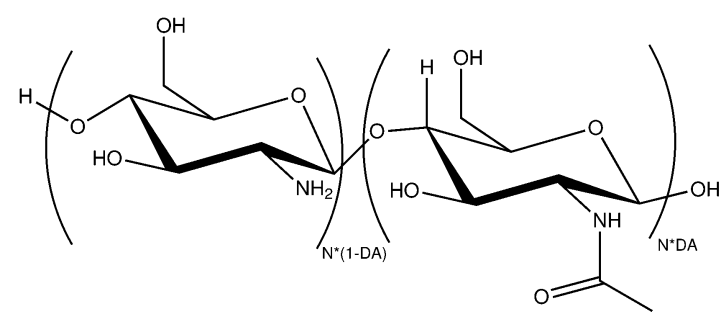

Fig. 9 Chemical structure of chitosan. generic properties of chitin and chitosan, ${ }^{\mathbf{1 3 2 - 1 3 5}}$ the most cited papers deal with chemical modifications of chitin and chitosan, ${ }^{\mathbf{1 3 6 - 1 3 9}}$ and the use of chitosan in medical applications, ${ }^{139-143}$ in food industry ${ }^{\mathbf{1 4 4 - 1 4 8}}$ and as a pollutant recoverer. ${ }^{149-155}$ These applications rely, of course, to a large extent on the pronounced biocompatibility of chitosan, which is also coupled to its antimicrobial properties. ${ }^{\mathbf{1 3 2 , 1 3 4 , 1 5 6}}$ Accordingly it is a very promising molecule for the use in more complex and hierarchically assembled systems. ${ }^{157}$

\subsection{Complex formation: structure and phase behaviour}

In the following we want to focus on work that has been done on complexes of chitosan and anionic surfactants. Quite a bit of effort has been dedicated to complexes formed by chitosan with sodium dodecyl sulphate (SDS). ${ }^{38,42,158-164}$ It was shown that SDS and chitosan form insoluble complexes well below the $\mathrm{cmc}$ of the surfactant and in a high polyelectrolyte excess, indicating a strong cooperative behavior ascribed to the hydrophobic interactions among the bound surfactant molecules. ${ }^{\mathbf{3 8 , 1 6 0}}$ In the work done by our group we also found that chitosans with different molecular weights and different degrees of acetylation (DA) (from ca. 2\% to $15 \%$ ) mixed with SDS, sodium dodecyl benzene sulphonate (SDBS) and sodium lauryl ether sulphate with an average of 2 ethylenoxide units per surfactant (SLES) form insoluble complexes over a wide range of mixing ratios and concentrations. ${ }^{165}$ The insoluble complexes formed by SDS and chitosan have been shown to exhibit marked Bragg peaks in the X-ray diffraction patterns, corresponding to a repeating distance of $c a .3 .7 \mathrm{~nm}$ which is characteristic of densely packed micelles (see Fig. 2b). ${ }^{\mathbf{1 5 8 , 1 6 1 , 1 6 6}}$

These insoluble complexes can be used for the formation of microcapsules. ${ }^{\mathbf{1 5 8 , 1 6 6}}$ In particular, if a chitosan solution is added to a SDS solution with a concentration above the cmc, capsules with approximately the size of the drop are formed. Much smaller droplets can be obtained if the added chitosan is emulsified in decane before being added to the SDS solution. ${ }^{\mathbf{1 6 6}}$ Rinaudo et al. showed that the wall thickness increases with the square root of time, indicating that the formation process is determined by interfacial diffusion of surfactant micelles and chitosan chains. They also determined the viscosity and elastic modulus of a $300 \mu \mathrm{m}$ thick layer to be $10^{6}$ to $10^{7} \mathrm{~Pa}$ s and $10^{4}$ to $10^{5} \mathrm{~Pa}$, respectively, ${ }^{166}$ the latter being comparable with that of high density polyethylene.

\subsection{Binding isotherms}

In a detailed thermodynamic study on the binding of SDS on chitosan, performed by Wei and Hudson, it was shown that the binding process could be well described with the Yang-Satake model only for chitosan with low DA. ${ }^{38}$ The authors found a value of $K \omega=2.23 \times 10^{4} \mathrm{M}^{-1}$ and $\omega=23$ for the binding to a chitosan with a DA of 0.08 and at $\mathrm{pH}=4.2$, which means that the binding is strongly cooperative. Interestingly, the addition of $\mathrm{NaBr}$ (up to $150 \mathrm{mM}$ ) shifts the binding of SDS to a higher surfactant content, due to electrostatic screening of the charges, but has almost no effect on the steepness of the binding isotherm, showing that the cooperative process is not affected by the presence of the salt, thereby indicating that the 
cooperative binding is mainly driven by hydrophobic interactions. Summarizing, the complexation of chitosan by SDS is a two-step process: first, single surfactant molecules bind to the polyelectrolyte chain due to electrostatic interactions; afterwards a strongly cooperative process takes place which is, in addition to electrostatics, characterized by strong hydrophobic interactions between the alkyl tails of the bound surfactant molecules. Accordingly, an increased ionic strength has an effect only in the first step of the binding process.

The binding of anionic surfactants to chitosan can be described with eqn (3) only for systems with low DA, i.e., $\beta<$ $0.2 .^{38}$ For higher values the model has to be corrected, taking into account the non-ionic and non-binding segments of $\mathrm{N}$ acetyl-glucosamine. For the corrected binding isotherm we refer to eqn (8) in ref. 38.

Thongngam and McClements investigated the influence of $\mathrm{pH}$, ionic strength and temperature on the binding of SDS to chitosan. ${ }^{42}$ The complex formation was studied at $\mathrm{pH}=3$ and 7 , chitosan being fully protonated and soluble in the former case, uncharged and insoluble in the latter. By use of isothermal titration calorimetry (ITC) and surfactant selective electrode (SSE) the authors showed that the binding of SDS on chitosan occurs only when the polyelectrolyte is charged to a sufficient extent, i.e., below $\mathrm{pH}=7$. However, the complex formed in acidic conditions cannot be redissolved with increasing $\mathrm{pH}$, indicating that on the experimental time scale irreversible complexes are formed. Furthermore, they could show that the amount of SDS bound to chitosan in acidic conditions is not influenced by changes of temperature $\left(10-50{ }^{\circ} \mathrm{C}\right)$ and ionic strength $(0-150 \mathrm{mmol} \mathrm{NaCl})$ and is $4-5$ mmol per gram of chitosan, which corresponds to a $1: 1$ complex (considering only the deacetylated units). Calorimetric titrations were performed at different temperatures, with the concentration of the surfactant in the titration syringe being above and below the cmc. The authors could show that surfactant aggregates are present in the complex, even at a total concentration of SDS below $0.2 \mathrm{mM}$, i.e., 40 times less than the cmc, and that the binding is driven by electrostatic interactions, with a $\Delta H_{\text {bind }}$ of $c a$. $6.5 \mathrm{~kJ} \mathrm{~mol}^{-1}$ SDS. Despite the interaction being mainly electrostatically driven, the addition of $100 \mathrm{mM} \mathrm{NaCl}$ at $\mathrm{pH}=3.0$ and $30^{\circ} \mathrm{C}$ has little effect on the binding of SDS.

In conclusion, the binding between SDS and chitosan is highly cooperative and the complex formation is a highly exothermic process. These complexes, once formed, cannot be redissolved with increasing $\mathrm{pH}$ or ionic strength, indicating that they are trapped in a deep energetic minimum. The very low solubility of the SDS-chitosan complexes, even at $Z \ll 1$ and $Z$ $\gg 1$, ratios where the polyelectrolyte should be virtually still fully charged, can be justified considering this minimum: when chitosan and surfactant solutions are mixed, a saturation of the polyelectrolyte takes place locally with the formation of an insoluble, electrically neutral complex, which does not redissolve on the experimental time scale.

\subsection{Interfacial aspects}

With respect to cellulose based systems, studies of interfacial properties of chitosan surfactant mixtures are more limited ${ }^{\mathbf{1 6 1 , 1 6 2 , 1 6 7 - 1 7 3}}$ and were recently reviewied. ${ }^{171}$ In the literature contradicting results on the surface activity of chitosan solutions can be found: while some authors state that chitosan itself shows no surface activity $\%,{ }^{\mathbf{1 6 1 , 1 6 8 , 1 6 9 , 1 7 4}}$ or report even an increase in surface tension up to $84 \mathrm{mN} \mathrm{m}^{-1}$ for $1 \%$ of $1.88 \mathrm{MDa}$ chitosan in a $0.1 \mathrm{M}$ acetic acid solution at $25{ }^{\circ} \mathrm{C},{ }^{174}$ on the contrary, other authors report a strong decrease in surface tension also for dilute chitosan solutions $\left(C_{\mathrm{p}} c a .0 .02 \%\right) .{ }^{171}$ However, the authors also report a very long adsorption induction time, i.e., the time before the surface tension starts to decrease, in the order of $10^{3}$ to $10^{4} \mathrm{~s}$, as to be expected for such a low molar concentration. In general, achieving equilibrium of the surface adsorption of high $M_{\mathrm{w}}$ polymers in aqueous solutions is problematic due to the very long equilibration times (which can easily go to days and months).

When chitosan is added to oppositely charged surfactant solutions, in most cases SDS, a lower surface tension with respect to the pure tenside solution is observed, with this effect being stronger for hydrophobically modified chitosan. ${ }^{167,168}$ This effect is explained by the formation of highly surface active SPECs, which form already at very low surfactant concentrations, where SDS alone shows no decrease of surface tension.

\subsection{Interactions with phospholipid vesicles}

Another system which recently has attracted the interest of the scientific community are mixtures of chitosan with phospholipid vesicles. ${ }^{\mathbf{1 6 9 , 1 7 5 - 1 8 6}}$ Briefly, chitosan adsorbs flat onto the surface of negatively, neutrally and positively charged vesicles $^{183,185}$ and the adsorption does not seem to be influenced by the vesicle size. ${ }^{183}$ However, as the studied vesicles were very large (LUVs of $200 \mathrm{~nm}$ and GUVs of some $\mu \mathrm{m}$ ) in comparison to the persistence length of chitosan, it is worth to suppose that only a "flat" surface is seen by the polyelectrolyte. When chitosan is added stepwise to a solution of negative LUVs one can observe a charge reversal from negative to positive and the formation of large clusters in proximity to charge neutrality. Finally, the addition of chitosan was shown not to affect the structure of the lipid bilayer ${ }^{\mathbf{1 8 4}}$ and to increase the stability of the membrane against $\mathrm{pH}$ and salt shocks. ${ }^{178}$ Inelastic neutron scattering showed that the presence of the chitosan drastically reduces the nanosecond dynamics of the phospholipid headgroup. ${ }^{\mathbf{1 8 6}}$

\subsection{Interactions with fatty acids}

Chitosan's ability to bind fatty acids and bile salts has been studied to some extent. ${ }^{\mathbf{1 7 0 , 1 8 7 - 1 9 1}}$ Due to the relevance of chitosan as a promising dietary supplement for antilipidemic treatments, such binding behavior has been investigated in acidic conditions, mimicking the stomach digestive conditions, and at neutral $\mathrm{pH}$, mimicking the duodenal intestinal tract. Even if carboxylic acids $\left(\mathrm{p} K_{\mathrm{a}} \sim 4.5\right)$ and chitosan $\left(\mathrm{p} K_{\mathrm{a}} \sim 6.5\right)$ are oppositely charged only in a narrow $\mathrm{pH}$ range, they form stable complexes throughout a large $\mathrm{pH}$ spectrum of 3-5. Under very acidic conditions $(\mathrm{pH}<3)$ chitosan is fully charged while the fatty acids are found in their protonated and therefore neutral forms and oil-in-water emulsions stabilized by chitosan are formed. ${ }^{189}$ 
In contrast, solid complexes between chitosan and sodium caprate have been reported to dissolve within one day in acidic, surfactant free solutions $(\mathrm{pH}=5$ and $10 \mathrm{mM} \mathrm{NaCl}) .{ }^{192}$ The authors could further show an enhancement of the stability of the solid complex when the surfactant is covalently coupled to the polyelectrolyte.

\section{Conclusions}

The complex formation in mixtures of oppositely charged biopolyelectrolytes and surfactants follows in general the behaviour observed for other, typically synthetic, polyelectrolytes. A main difference is that basically all biopolyelectrolytes have a rather high intrinsic persistence length (apart from the stiffening that may result from the electrostatic charging) compared to many synthetic polyelectrolytes (for instance polyacrylates). This high stiffness also applies to the biopolyelectrolytes that are in the focus of this review: ionically modified cellulose and chitosan. Therefore the structures formed in their complexes have more likely a tendency for an extended conformation of the polyelectrolytes but nonetheless normally lead to precipitation around the equimolar charge ratio between polyelectrolytes and surfactants.

The structure of the surfactant aggregates in these complexes is typically not much affected by the presence of the biopolyelectrolyte but mostly determined by the packing conditions of the surfactant molecules. Accordingly the binding of the surfactant to the polyelectrolytes is generally highly cooperative. Of course, for highly curved surfactant surfaces, such as in spherical micelles, it becomes difficult for the rather stiff biopolyelectrolytes to follow this curvature and the interaction here and the structures formed are often quite different from those in the case of highly flexible polyelectrolytes. Despite these restrictions the structural variety of the complexes formed is very rich and different structural entities can be created. For instance, for the case of achieving cylindrical micellar aggregates and sufficiently long polyelectrolytes one may form highly viscous homogeneous solutions already for relatively low concentrations thereby allowing for a simple rheological control over many orders of viscosity by relatively small changes of surfactant concentration.

By balancing the interactions one may also form complexes with finite sizes in the range of $50-500 \mathrm{~nm}$, which can be interesting delivery systems as they contain conventional hydrophobic domains (in the micellar cores), water-insoluble complex domains, and water-soluble polyelectrolyte chains. This renders them interesting objects for the purposes of selective solubilisation, even allowing for different types of solubilisation sites within one self-assembled aggregate.

These properties together with the fact that they are ecologically favourable and typically highly biocompatible make these biopolyelectrolytes a very interesting alternative to conventional polyelectrolytes, which due to their relatively high chain stiffness allow for the formation of complex structures not so easily formed by other polyelectrolytes. In addition, the described biopolyelectrolytes chitosan and modified cellulose are easily available in large quantities. Therefore one can expect an increasing use in a large variety of applied formulations that will employ these specific properties of the biopolyelectrolyte interactions with surfactants.

\section{Acknowledgements}

We gratefully acknowledge financial support of this work by the BMBF project 05K10KT1. The authors would like to thank the ILL for granted beam time. IH wishes to thank E. Hirtenlechner for fruitful discussions.

\section{References}

1 K. Holmberg, B. Jönsson, B. Kronberg and B. Lindman, Surfactants and Polymers in Aqueous Solution, John Wiley \& Sons, Ltd, Chichester, UK, 2002.

2 Polymer-Surfactant Systems, ed. J. C. T. Kwak, Marcel Dekker, Inc., 1998, vol. 77.

3 A. V. Kabanov and A. B. Zezin, Pure Appl. Chem., 1984, 56, 343-354.

4 A. J. Konop and R. H. Colby, Langmuir, 1999, 15, 58-65.

5 E. D. Goddard, J. Am. Oil Chem. Soc., 1994, 71, 1-16.

6 K. T. Oh, T. K. Bronich, L. Bromberg, T. A. Hatton and A. V. Kabanov, J. Controlled Release, 2006, 115, 9-17.

7 C. Wang and K. C. Tam, J. Phys. Chem. B, 2004, 108, 89768982.

8 B. Magny, I. Iliopoulos, R. Zana and R. Audebert, Langmuir, 1994, 10, 3180-3187.

9 P. S. Leung and E. D. Goddard, Langmuir, 1991, 7, 608-609.

10 T. K. Bronich, A. Nehls, A. Eisenberg and A. V. Kabanov, Colloids Surf., B, 1999, 16, 243-251.

11 S. V. Vinogradov, T. K. Bronich and A. V. Kabanov, Adv. Drug Delivery Rev., 2002, 54, 135-147.

12 T. Nylander, Y. Samoshina and B. Lindman, Adv. Colloid Interface Sci., 2006, 123, 105-123.

13 F. Aloulou, S. Boufi and D. Beneventi, J. Colloid Interface Sci., 2004, 280, 350-358.

14 I. Hoffmann, M. Theile, S. Grätz, J. Scholz, P. Barreleiro, W. von Rybinski and M. Gradzielski, Langmuir, 2012, 28, 11400-11409.

15 G. S. Manning, J. Chem. Phys., 1969, 51, 924.

16 M. Burkhardt, M. Ruppel, S. Tea, M. Drechsler, R. Schweins, D. V. Pergushov, M. Gradzielski, A. B. Zezin and A. H. E. Müller, Langmuir, 2008, 24, 1769-1777.

17 S. Zhou, C. Burger, F. Yeh and B. Chu, Macromolecules, 1998, 31, 8157-8163.

18 T. K. Bronich, A. M. Popov, A. Eisenberg, V. A. Kabanov and A. V. Kabanov, Langmuir, 2000, 16, 481-489.

19 J.-F. Berret, P. Hervé, O. Aguerre-Chariol and J. Oberdisse, J. Phys. Chem. B, 2003, 107, 8111-8118.

20 M. Uchman, M. Štěpánek, S. Prévost, B. Angelov, J. Bednár, M.-S. Appavou, M. Gradzielski and K. Procházka, Macromolecules, 2012, 45, 6471-6480.

21 J. Skolnick and M. Fixman, Macromolecules, 1977, 10, 944948.

22 T. Salditt, I. Koltover, J. Rädler and C. Safinya, Phys. Rev. Lett. , 1997, 79, 2582-2585. 
23 Q. Cao, C. Zuo, L. Li and M. Gao, Colloid Polym. Sci., 2011, 289, 1089-1102.

24 N. Beheshti, K. Zhu, A.-L. Kjøniksen and B. Nyström, Colloid Polym. Sci., 2010, 288, 1121-1130.

25 M. Almgren, P. Hansson, E. Mukhtar and J. Van Stam, Langmuir, 1992, 8, 2405-2412.

26 K. Thalberg, B. Lindman and K. Bergfeldt, Langmuir, 1991, 7, 2893-2898.

27 K. Pojják, E. Bertalanits and R. Mészáros, Langmuir, 2011, 27, 9139-9147.

28 P. F. C. Lim, L. Y. Chee, S. B. Chen and B.-H. Chen, J. Phys. Chem. B, 2003, 107, 6491-6496.

29 N. Plucktaveesak, A. J. Konop and R. H. Colby, J. Phys. Chem. $B, 2003,107,8166-8171$.

30 Q. Wu, M. Du, T. Ye, Y.-G. Shangguan, J.-P. Zhou and Q. Zheng, Colloid Polym. Sci., 2009, 287, 911-918.

31 P. M. Claesson, M. Bergström, A. Dedinaite, M. Kjellin, J.-F. Legrand and I. Grillo, J. Phys. Chem. B, 2000, 104, 11689-11694.

32 S. Zhou and B. Chu, Adv. Mater., 2000, 12, 545-556.

33 L. M. Bergström, U. R. M. Kjellin, P. M. Claesson and I. Grillo, J. Phys. Chem. B, 2004, 108, 1874-1881.

34 I. Satake and J. T. Yang, Biopolymers, 1976, 15, 2263-2275. 35 B. H. Zimm and J. K. Bragg, J. Chem. Phys., 1959, 31, 526.

36 T. Nishio and T. Shimizu, Biophys. Chem., 2005, 117, 19-25. 37 T. Nishio, T. Shimizu, J. C. T. Kwak and A. Minakata, Biophys. Chem., 2003, 104, 501-508.

38 Y. C. Wei and S. M. Hudson, Macromolecules, 1993, 26, 4151-4154.

39 S. Kosmella, J. Koetz, K. Shirahama and J. Liu, J. Phys. Chem. $B$, 1998, 102, 6459-6464.

40 H. Fukui, I. Satake and K. Hayakawa, Langmuir, 2002, 18, 4465-4470.

41 C. F. J. Faul and M. Antonietti, Chemistry, 2002, 8, 27642768.

42 M. Thongngam and D. J. Mcclements, Langmuir, 2005, 21, 79-86.

43 G. Li, H. Ma and J. Hao, Soft Matter, 2012, 8, 896.

44 D. Li, M. S. Kelkar and N. J. Wagner, Langmuir, 2012, 28, 10348-10362.

45 Y. Lapitsky, M. Parikh and E. W. Kaler, J. Phys. Chem. B, 2007, 111, 8379-8387.

46 L. Chiappisi, D. Li, N. J. Wagner and M. Gradzielski, J. Phys. Chem. B, submitted.

47 A. B. Kayitmazer, D. Shaw and P. L. Dubin, Macromolecules, 2005, 38, 5198-5204.

48 V. A. Izumrudov, Russ. Chem. Rev., 2008, 77, 401-415.

49 V. A. Izumrudov, in Advances in Regenerative Medicine: Role of Nanotechnology, and Engineering Principles, Springer Netherlands, 2010, ch. 18, pp. 379-405.

50 T. Heinze and T. Liebert, Prog. Polym. Sci., 2001, 26, 1689-1762. 51 L.-M. Zhang, Macromol. Mater. Eng., 2001, 286, 267-275.

52 T. Heinze, A. Koschella, L. Magdaleno-Maiza and A. S. Ulrich, Polym. Bull., 2001, 46, 7-13.

53 T. Heinze, M. Nikolajski, S. Daus, T. M. D. Besong, N. Michaelis, P. Berlin, G. A. Morris, A. J. Rowe and S. E. Harding, Angew. Chem., Int. Ed., 2011, 50, 8602-8604.
54 K. Rahn, M. Diamantoglou, D. Klemm, H. Berghmans and T. Heinze, Angew. Makromol. Chem., 1996, 238, 143-163.

55 C. S. P. Zarth, A. Koschella, A. Pfeifer, S. Dorn and T. Heinze, Cellulose, 2011, 18, 1315-1325.

56 T. Genco, L. F. Zemljič, M. Bračič, K. Stana-Kleinschek and T. Heinze, Macromol. Chem. Phys., 2012, 213, 539-548.

57 T. Heinze, T. Genco, K. Petzold-Welcke and H. Wondraczek, Cellulose, 2012, 19, 1305-1313.

58 M. Miyake and Y. Kakizawa, Int. J. Cosmet. Sci., 2010, 32, 473. 59 F. J. C. Fité, J. Text. Inst., 1993, 84, 582-592.

60 F. Jost, H. Andree and M. J. Schwuger, Colloid Polym. Sci., 1986, 264, 56-64.

61 F. M. Winnik and S. T. Regismond, Colloids Surf., A, 1996, 118, 1-39.

62 P. Hansson and B. Lindman, Curr. Opin. Colloid Interface Sci., 1996, 1, 604-613.

63 L. Piculell and B. Lindman, Adv. Colloid Interface Sci., 1992, 41, 149-178.

64 L. Piculell, J. Norrman, A. V. Svensson, I. Lynch, J. S. Bernardes and W. Loh, Adv. Colloid Interface Sci., 2009, 147-148, 228-236.

65 D. Wever, F. Picchioni and A. Broekhuis, Prog. Polym. Sci., 2011, 36, 1558-1628.

66 E. D. Goddard, J. Colloid Interface Sci., 2002, 256, 228-235.

67 C. D. Bain, P. M. Claesson, D. Langevin, R. Meszaros, T. Nylander, C. Stubenrauch, S. Titmuss and R. von Klitzing, Adv. Colloid Interface Sci., 2010, 155, 32-49.

68 D. Taylor, R. Thomas and J. Penfold, Adv. Colloid Interface Sci., 2007, 132, 69-110.

69 B. Nyström, A.-L. Kjøniksen, N. Beheshti, K. Zhu and K. D. Knudsen, Soft Matter, 2009, 5, 1328-1339.

70 E. D. Goddard and R. B. Hannan, J. Colloid Interface Sci., 1976, 55, 73-79.

71 U. Kästner, H. Hoffmann, R. Donges and R. Ehrler, Colloids Surf., A, 1996, 112, 209-225.

72 S. Zhou, C. Xu, J. Wang, P. Golas, J. Batteas and L. Kreeger, Langmuir, 2004, 20, 8482-8489.

73 A. Svensson, J. Sjöström, T. Scheel and L. Piculell, Colloids Surf., A, 2003, 228, 91-106.

74 S. Dos Santos, C. Gustavsson, C. Gudmundsson, P. Linse and L. Piculell, Langmuir, 2010, 27, 592-603.

75 S. Trabelsi, S. Guillot, E. Raspaud, M. Delsanti, D. Langevin and F. Boué, Adv. Mater., 2006, 18, 2403-2406.

76 I. Hoffmann, P. Heunemann, S. Prévost, R. Schweins, N. J. Wagner and M. Gradzielski, Langmuir, 2011, 27, 4386-4396.

77 I. Hoffmann, S. Prévost, M. Medebach, S. E. Rogers, N. J. Wagner and M. Gradzielski, Tenside, Surfactants, Deterg., 2011, 48, 488-494.

78 E. F. Marques, O. Regev, A. Khan, M. D. G. Miguel and B. Lindman, Macromolecules, 1999, 32, 6626-6637.

79 Y. Yamaguchi, Y. Inaba, H. Uchiyama and H. Kunieda, Colloid Polym. Sci., 1999, 277, 1117-1124.

80 D. Langevin, Adv. Colloid Interface Sci., 2009, 147-148, 170177.

81 J. Liu, Q. Zhang, Y. Huo, M. Zhao, D. Sun, X. Wei, S. Liu and L. Zheng, Colloid Polym. Sci., 2012, 290, 1721-1730. 
82 J. Sjöström and L. Piculell, Colloids Surf., A, 2001, 183-185, 429-448.

83 S. Guillot, D. Mcloughlin, N. Jain, M. Delsanti and D. Langevin, J. Phys.: Condens. Matter, 2003, 15, S219-S224.

84 M. Tsianou, A. L. Kjøniksen, K. Thuresson and B. Nyström, Macromolecules, 1999, 32, 2974-2982.

85 N. Beheshti, G. T. M. Nguyen, A. L. Kjøniksen, K. D. Knudsen and B. Nyström, Colloids Surf., A, 2006, 279, 40-49.

86 N. Jain, S. Trabelsi, S. Guillot, D. Mcloughlin, D. Langevin, P. Letellier and M. Turmine, Langmuir, 2004, 20, 8496-8503.

87 L. G. Patruyo, A. J. Muller and A. E. Saez, Polymer, 2002, 43, 6481-6493.

88 Q. Wu, Y. Shangguan, M. Du, J. Zhou, Y. Song and Q. Zheng, J. Colloid Interface Sci., 2009, 339, 236-242.

89 N. Beheshti, A.-L. Kjøniksen, K. Zhu, K. D. Knudsen and B. Nyström, J. Phys. Chem. B, 2010, 114, 6273-6280.

90 M. Tsianou and P. Alexandridis, Langmuir, 1999, 15, 81058112.

91 K. L. Ngai, A. K. Rajagopal and S. Teitler, J. Chem. Phys., 1988, 88, 5086-5094.

92 K. L. Ngai, A. K. Rajagopal and T. P. Lodge, J. Polym. Sci., Part B: Polym. Phys., 1990, 28, 1367-1377.

93 K. L. Ngai, Adv. Colloid Interface Sci., 1996, 64, 1-43.

94 K. S. Schweizer, M. Fuchs, G. Szamel, M. Guenza and H. Tang, Macromol. Theory Simul., 1997, 6, 1037-1117.

95 K. Kroy and E. Frey, Phys. Rev. E: Stat., Nonlinear, Soft Matter Phys., 1997, 55, 3092-3101.

96 E. Bartsch, M. Antonietti, W. Schupp and H. Sillescu, J. Chem. Phys., 1992, 97, 3950-3963.

97 B. Nyström and B. Lindman, Macromolecules, 1995, 28, 967974.

98 A. L. Kjøniksen, S. Nilsson, K. Thuresson, B. Lindman and B. Nyström, Macromolecules, 2000, 33, 877-886.

99 N. Beheshti, H. Bu, K. Z. Zhu, A. L. Kjøniksen, K. D. Knudsen, R. Pamies, J. G. H. Cifre, J. G. de La Torre and B. Nyström, J. Phys. Chem. B, 2006, 110, 6601-6608.

100 J. Merta, V. M. Garamus, R. Willumeit and P. Stenius, Langmuir, 2002, 18, 7272-7278.

101 J. Merta, V. M. Garamus, A. I. Kuklin, R. Willumeit and P. Stenius, Langmuir, 2000, 16, 10061-10068.

102 J. Han, F. Cheng, X. Wang and Y. Wei, Carbohydr. Polym., 2012, 88, 139-145.

103 S. Nilsson, K. Thuresson, P. Hansson and B. Lindman, J. Phys. Chem. B, 1998, 102, 7099-7105.

104 S. Guillot, M. Delsanti, S. Desert and D. Langevin, Langmuir, 2003, 19, 230-237.

105 Y. Lapitsky and E. W. Kaler, Soft Matter, 2006, 2, 779-784.

106 Y. Lapitsky, W. J. Eskuchen and E. W. Kaler, Langmuir, 2006, 22, 6375-6379.

107 K. Procházka, Z. Limpouchová, F. Uhlík, P. Košovan, P. Matějíček, M. Štěpánek, M. Uchman, J. Kuldová, R. Šachl, J. Humpolíčkova and M. Hof, Adv. Polym. Sci., 2010, 241, 187-249.

108 A. N. de Belder and K. Granath, Carbohydr. Res., 1973, 30, 375-378.

109 F. M. Winnik, M. A. Winnik, S. Tazuke and C. K. Ober, Macromolecules, 1987, 20, 38-44.
110 F. M. Winnik, S. T. A. Regismond and E. D. Goddard, Langmuir, 1997, 13, 111-114.

111 I. Hoffmann, C. Oppel, U. Gernert, P. Barreleiro, W. von Rybinski and M. Gradzielski, Langmuir, 2012, 28, 7695-7703.

112 S. T. A. Regismond, Y.-M. Heng, E. D. Goddard and F. M. Winnik, Langmuir, 1999, 15, 3007-3010.

113 A. Das Burman, T. Dey, B. Mukherjee and A. R. Das, Langmuir, 2000, 16, 10020-10027.

114 K. Ohbu, O. Hiraishi and I. Kashiwa, J. Am. Oil Chem. Soc., 1982, 59, 108-112.

115 Y. Lapitsky, M. Parikh and E. W. Kaler, J. Phys. Chem. B, 2007, 111, 8379-8387.

116 S. Trabelsi and D. Langevin, Langmuir, 2007, 23, 1248-1252. 117 M. Barck and P. Stenius, Colloids Surf., A, 1994, 89, 59-69.

118 S. T. A. Regismond, F. M. Winnik and E. D. Goddard, Colloids Surf., A, 1996, 119, 221-228.

119 S. T. A. Regismond, K. D. Gracie, F. M. Winnik and E. D. Goddard, Langmuir, 1997, 13, 5558-5562.

120 D. Zimin, V. S. J. Craig and W. Kunz, Langmuir, 2004, 20, 2282-2291.

121 D. Zimin, V. S. J. Craig and W. Kunz, Langmuir, 2004, 20, 8114-8123.

122 E. Terada, Y. Samoshina, T. Nylander and B. Lindman, Langmuir, 2004, 20, 1753-1762.

123 E. Terada, Y. Samoshina, T. Nylander and B. Lindman, Langmuir, 2004, 20, 6692-6701.

124 A. V. Svensson, L. Huang, E. S. Johnson, T. Nylander and L. Piculell, ACS Appl. Mater. Interfaces, 2009, 1, 2431-2442.

125 A. V. Svensson, E. S. Johnson, T. Nylander and L. Piculell, ACS Appl. Mater. Interfaces, 2010, 2, 143-156.

126 S. Kundu, J. Colloid Interface Sci., 2010, 344, 547-555.

127 M. Clauzel, E. S. Johnson, T. Nylander, R. K. Panandiker, M. R. Sivik and L. Piculell, ACS Appl. Mater. Interfaces, 2011, 3, 2451-2462.

128 L. Fras-Zemljič, P. Stenius, J. Laine and K. StanaKleinschek, Cellulose, 2006, 13, 655-663.

129 I. Hoffmann, C. Oppel, S. Prévost, U. Gernert, P. Barreleiro, W. von Rybinski and M. Gradzielski, Colloids Surf., B, 2012, 91, 175-180.

130 M. E. Zakrzewska, E. Bogel-Łukasik and R. Bogel-Łukasik, Energy Fuels, 2010, 24, 737-745.

131 T. R. Dawsey and C. L. Mccormick, J. Macromol. Sci., Part C: Polym. Rev., 1990, 30, 405-440.

132 M. N. V. R. Kumar, React. Funct. Polym., 2000, 46, 1-27.

133 F. Shahidi and R. Abuzaytoun, Adv. Food Nutr. Res., 2005, 49, 93-135.

134 M. Rinaudo, Prog. Polym. Sci., 2006, 31, 603-632.

$135 \mathrm{~S}$. Tokura and H. Tamura, Comprehensive Glycoscience, 2007, ch. 2.14, pp. 449-475.

136 W. Sajomsang, Carbohydr. Polym., 2010, 80, 631-647.

137 K. Harish Prashanth and R. Tharanathan, Trends Food Sci. Technol., 2007, 18, 117-131.

138 V. Mourya and N. Inamdar, React. Funct. Polym., 2008, 68, 1013-1051.

139 M. N. V. R. Kumar, R. A. A. Muzzarelli, C. Muzzarelli, H. Sashiwa and A. J. Domb, Chem. Rev., 2004, 104, 60176084. 
140 O. Felt, P. Buri and R. Gurny, Drug Dev. Ind. Pharm., 1998, 24, 979-993.

141 S. Senel and S. J. Mcclure, Adv. Drug Delivery Rev., 2004, 56, 1467-1480.

142 J. K. Suh, H. W. T. Matthew and J.-K. Francis Suh, Biomaterials, 2000, 21, 2589-2598.

143 R. A. Muzzarelli, Carbohydr. Polym., 2009, 76, 167-182.

144 F. Shahidi, Trends Food Sci. Technol., 1999, 10, 37-51.

145 H. K. No, S. P. Meyers, W. Prinyawiwatkul and Z. Xu, J. Food Sci., 2007, 72, R87-R100.

146 P. Dutta, S. Tripathi, G. Mehrotra and J. Dutta, Food Chem., 2009, 114, 1173-1182.

147 M. Aider, LWT-Food Sci. Technol., 2010, 43, 837-842.

148 A. Alishahi and M. Aïder, Food Bioprocess Technol., 2011, 5, 817-830.

149 S. E. Bailey, T. J. Olin, R. M. Bricka and D. D. Adrian, Water Res., 1999, 33, 2469-2479.

150 P. Miretzky and A. F. Cirelli, J. Hazard. Mater., 2009, 167, 10-23.

151 H. K. No and S. P. Meyers, Rev. Environ. Contam. Toxicol., 2000, 163, 1-28.

152 C. Gerente, V. K. C. Lee, P. L. Cloirec and G. Mckay, Crit. Rev. Environ. Sci. Technol., 2007, 37, 41-127.

153 E. Onsosyen, O. Skaugrud and E. Onsø Yen, J. Chem. Technol. Biotechnol., 1990, 49, 395-404.

154 E. Guibal, Sep. Purif. Technol., 2004, 38, 43-74.

155 R. A. Muzzarelli, Carbohydr. Polym., 2011, 84, 54-63.

156 B. K. Park and M.-M. Kim, Int. J. Mol. Sci., 2010, 11, 51525164.

157 G. F. Payne and S. R. Raghavan, Soft Matter, 2007, 3, 521527.

158 V. Babak, E. A. Merkovich, J. Desbrières and M. Rinaudo, Polym. Bull., 2000, 45, 77-81.

159 A. G. S. Prado, J. L. Macedo, S. C. L. Dias and J. A. Dias, Colloids Surf., B, 2004, 35, 23-27.

160 M. Thongngam and D. J. Mcclements, J. Agric. Food Chem., 2004, 52, 987-991.

161 M. Lundin, L. Macakova, A. Dedinaite and P. M. Claesson, Langmuir, 2008, 24, 3814-3827.

162 C. Onesippe and S. Lagerge, Colloids Surf., A, 2008, 317, 100-108.

163 H. Bao, L. Li, L. H. Gan and H. Zhang, Macromolecules, 2008, 41, 9406-9412.

164 Y.-W. Wang, N.-B. Li and H.-Q. Luo, Luminescence, 2008, 23, 126-131.

165 L. Chiappisi, personal communication, 2012.

166 M. Rinaudo, N. R. Kildeeva and V. Babak, Russ. J. Gen. Chem., 2008, 78, 2239-2246.

167 C. Onésippe and S. Lagerge, Colloids Surf., A, 2008, 330, 201-206.
168 V. Babak, I. Lukina, G. Vikhoreva, J. Desbrières and M. Rinaudo, Colloids Surf., A, 1999, 147, 139-148.

169 F. J. Pavinatto, A. Pavinatto, L. Caseli, D. S. D. Santos, T. M. Nobre, M. E. D. Zaniquelli and O. N. Oliveira, Biomacromolecules, 2007, 8, 1633-1640.

170 H. Parra-Barraza, M. G. Burboa, M. Sánchez-Vázquez, J. Juárez, F. M. Goycoolea and M. A. Valdez, Biomacromolecules, 2005, 6, 2416-2426.

171 J. Desbrières and V. G. Babak, Polym. Int., 2006, 55, 11771183.

172 J. Desbrières and V. Babak, Soft Matter, 2010, 6, 2358.

173 M. Z. Elsabee, R. E. Morsi and A. M. Al-Sabagh, Colloids Surf., B, 2009, 74, 1-16.

174 G. Qun and W. Ajun, Carbohydr. Polym., 2006, 64, 29-36.

175 I. Henriksen, G. Smistad and J. Karlsen, Int. J. Pharm., 1994, 101, 227-236.

176 I. Henriksen, S. R. Våagen, S. A. Sande, G. Smistad and J. Karlsen, Int. J. Pharm., 1997, 146, 193-203.

177 F. J. Pavinatto, L. Caseli, A. Pavinatto, D. S. Dos Santos, T. M. Nobre, M. E. D. Zaniquelli, H. S. Silva, P. B. Miranda and O. N. de Oliveira, Langmuir, 2007, 23, 7666-7671.

178 F. Quemeneur, A. Rammal, M. Rinaudo and B. PépinDonat, Biomacromolecules, 2007, 8, 2512-2519.

179 F. Quemeneur, M. Rinaudo and B. Pépin-Donat, Biomacromolecules, 2008, 9, 2237-2243.

180 F. Quemeneur, M. Rinaudo and B. Pépin-Donat, Biomacromolecules, 2008, 9, 396-402.

181 O. Mertins, N. P. Da Silveira, A. R. Pohlmann, A. P. Schröder and C. M. Marques, Biophys. J., 2009, 96, 2719-2726.

182 O. Mertins, P. H. Schneider, A. R. Pohlmann and N. P. Da Silveira, Colloids Surf., B, 2010, 75, 294-299.

183 F. Quemeneur, M. Rinaudo, G. Maret and B. Pépin-Donat, Soft Matter, 2010, 6, 4471.

184 Y. Gerelli, M. T. Di Bari, A. Deriu, D. Clemens and L. Almásy, Soft Matter, 2010, 6, 2533.

185 O. Mertins and R. Dimova, Langmuir, 2011, 27, 5506-5515.

186 Y. Gerelli, V. G. Sakai, J. Ollivier and A. Deriu, Soft Matter, 2011, 7, 3929-3935.

187 J. L. Nauss, J. L. Thompson and J. Nagyvary, Lipids, 1983, 18, 714-719.

188 D. W. S. Wong, F. A. Gastineau, K. S. Gregorski, S. J. Tillin and A. E. Pavlath, J. Agric. Food Chem., 1992, 40, 540-544.

189 M. S. Rodríguez and L. E. Albertengo, Biosci., Biotechnol., Biochem., 2005, 69, 2057-2062.

190 P. Wydro, B. Krajewska and K. Hac-Wydro, Biomacromolecules, 2007, 8, 2611-2617.

191 K. Kofuji, M. Nakamura, T. Isobe, Y. Murata and S. Kawashima, Food Chem., 2008, 109, 167-171.

192 A. J. Worthen and Y. Lapitsky, Colloid Polym. Sci., 2011, 289, 1589-1596. 